\title{
Activation of microglia bolsters synapse formation
}

\author{
Gonçalo Cristovão ${ }^{1 \dagger}$, Maria J. Pinto ${ }^{1,2+}$, Rodrigo A. Cunha ${ }^{1,3}$, Ramiro D. Almeida $^{1}$ and \\ Catarina A. Gomes ${ }^{1,4}$ * \\ ${ }^{1}$ CNC-Center for Neuroscience and Cell Biology, University of Coimbra, Coimbra, Portugal \\ 2 PhD Programme in Experimental Biology and Biomedicine (PDBEB), Center for Neuroscience and Cell Biology, University of Coimbra, Coimbra, Portugal \\ ${ }^{3}$ Faculty of Medicine, Biochemistry, University of Coimbra, Coimbra, Portugal \\ ${ }^{4}$ Faculty of Medicine, Pharmacology and Experimental Therapeutics, University of Coimbra, Coimbra, Portugal \\ *Correspondence: catarinareisvalegomes@gmail.com
}

Edited by:

Raquel Ferreira, University of Southern California, USA

Reviewed by:

Tiago Santos, University of Beira Interior, Portugal

${ }^{t}$ These authors have contributed equally to this work.

Keywords: brain wiring, microglia, neurodevelopment, neuroinflammation, pre-synaptic differentiation, synapse formation

\section{A commentary on}

Brain inflammation induces postsynaptic changes during early synapse formation in adult-born hippocampal neurons

by Chugh, D., Nilsson, P., Afjei, S. A., Bakochi, A., and Ekdahl, C. T. (2013). Exp. Neurol. 250, 176-188. doi: 10.1016/ j.expneurol.2013.09.005

Microglial cells in the central nervous system (CNS) are major players of innate immunity, the first line of defense in the presence of danger signals, from bacterial infections to mediators released by neurons upon cytotoxic insult (Kettenmann et al., 2011). Particular attention has been paid to the involvement of microglia in pathological conditions and their eventual dual role, as disease amplifiers and/or executors of brain repair, is still a matter of controversy (Benarroch, 2013); the fact that inflammation is itself a strategy to contain biological threats, which inevitably leads to variable degrees of damage, may explain the apparent duality of roles attributed to microglia. These microglial functions are mainly dependent on the release of a plethora of mediators (from trophic factors to anti- and pro-inflammatory cytokines or chemokines) and are accompanied by characteristic changes of morphology, ranging from highly ramified to amoeboid shapes (Kettenmann et al., 2011).

Although the role of microglia has been mostly approached from the angle of pathology, microglial cells are also active players in healthy conditions by constantly monitoring the brain parenchyma (Davalos et al., 2005; Nimmerjahn et al., 2005) and correcting deviations from homeostasis; accordingly, several studies reported that the genetic alteration of molecules selectively blunting specific microglial functions impacted on synaptic transmission and synaptic plasticity (Pascual et al., 2012; Ji et al., 2013; Zhang et al., 2014), leading to deficits of behavioral response, such as social interaction, motor learning, or short-term memory (Rogers et al., 2011; Parkhurst et al., 2013; Zhan et al., 2014). These functional effects of microglia on synaptic plasticity and the direct observation of physical apposition of microglia processes with synapses led to the emergence of the concept that microglia could directly interact with synapses (Wake et al., 2009; Tremblay et al., 2010), forming the so called quad-partite synapses (Schafer et al., 2013). This was re-enforced by the observed ability of microglia to engulf and remove synapses, a process named synaptic pruning, which is crucial both for adequate synaptic wiring during neurodevelopment, as well as during synaptic re-wiring following brain injury (Wake et al., 2009; Schafer et al., 2012; Bialas and Stevens, 2013; reviewed in Kettenmann et al., 2013).

The recent report of Chugh et al. (2013) provides evidence supporting a more complex role for microglia-associated neuroinflammation in the process of synaptic wiring. In fact, this report shows that the creation of an inflammatory environment in the hippocampus of adult mice triggers a region-selective increase in the number of thin dentritic spines endowed with PSD-95, indicative of enhanced synaptic connectivity of newborn neurons, without overt changes in neuronal or astrocytic morphology. This is in striking agreement with other reports showing that the functional impairment of specific microglia functions or the ablation of microglia triggered an increase of excitatory synaptic transmission (Pascual et al., 2012; Ji et al., 2013).

However, it remained to be shown if this ability of microglia to bolster synapse formation resulted from a direct signaling of microglia onto maturating synapses and there is still no information about the putative ability of microglia to control the axonal sprouting. This led us to design an experimental protocol to study the direct interaction between microglia and immature axon/pre-synaptic terminals. This was achieved using microfluidic chambers where hippocampal neurons are plated in one compartment and, as the neurons develop, the axons grow through the microgrooves to reach a second physically isolated compartment (Taylor et al., 2005; Neto et al., 2014). The growing axons, but not the cell bodies, were exposed to the N9 microglia cell line, which was previously primed with lipopolysaccharide (LPS), a "classic" microglia activator. When we measured the density of nerve terminals by immunofluorescence against 


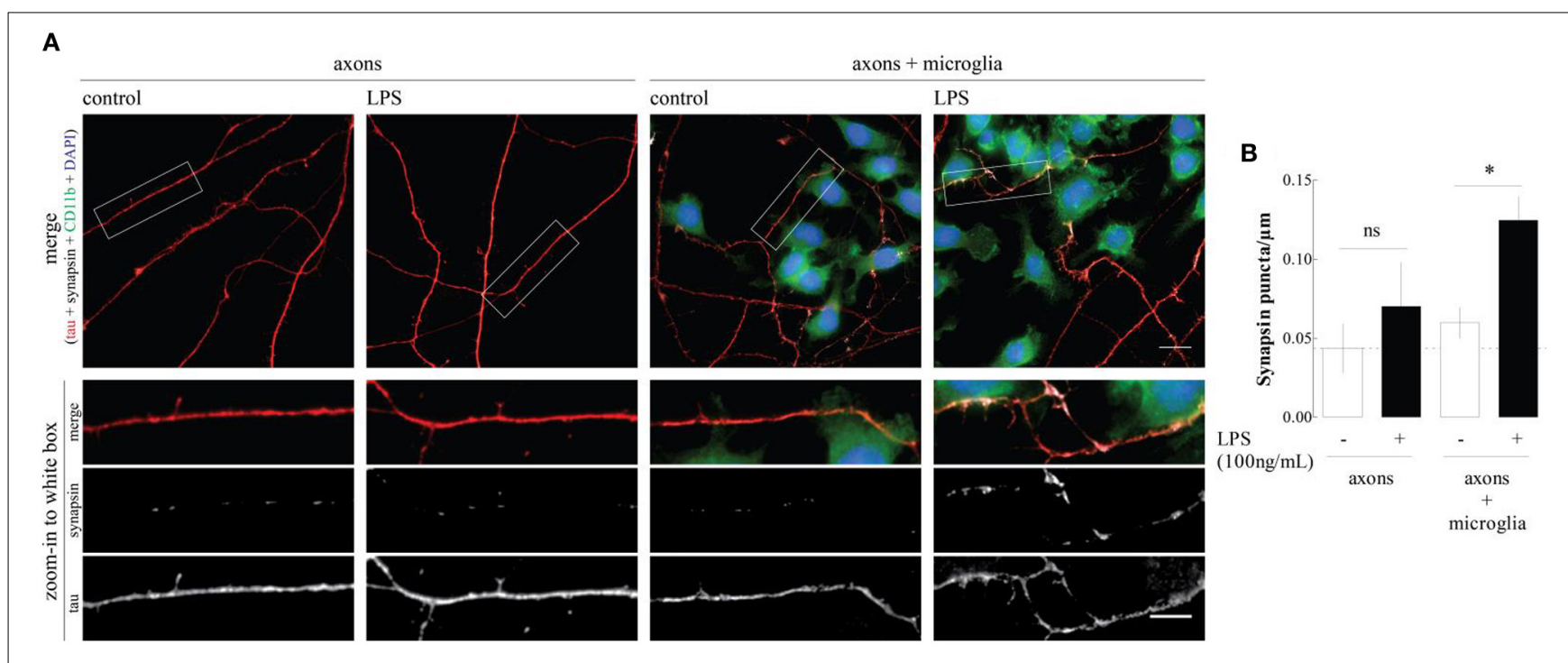

FIGURE 1 | LPS-primed microgial cells increase synapsin puncta along axons. (A) Representative images of the axonal/microglia compartment of microfluidic chambers in which rat embryo hippocampal neurons were allowed to grow for 7 days. When indicated, before axons arrival to the axonal compartment a microglia cell line was cultured (DIV3) and activated with LPS for $6 \mathrm{~h}$ (DIV4). Cultures were stained for CD11b (microglia marker, green),
DAPI (nuclei, blue), tau (axonal marker, red), and synapsin I (synaptic vesicles marker, white). Scale bars are 10 and $5 \mu \mathrm{m}$ for upper and lower zoom-in images, respectively. (B) Quantification of the number of synapsin puncta per axonal length. Results, obtained analyzing 20 images per condition, are expressed as mean \pm s.e.m. of three independent experiments, $\left(^{*} p<0.5\right.$, by Two-Way ANOVA followed by Bonferroni post-hoc test). the pre-synaptic marker synapsin I, we found that activated microglia caused a clear-cut increase in the axonal density of the protein, which was not observed in the presence on non-primed microglia or in isolated axons (Figure 1). Recently, Parkhurst et al. (2013) demonstrated that microglia regulates synapse formation through brain-derived neurotrophic factor (BDNF), which is in line with our previous work (Gomes et al., 2013), showing the ability of LPS to increase BDNF release by $\mathrm{N} 9$ microglial cells. The increase in the number of synapsin clusters is, however, more striking in axons which are in contact with microglial cells, suggesting that a cell adhesion molecule might also mediate this effect (inset, Figure 1). Further studies using conditioned medium from LPS-primed microglial cells may help clarify the relative contribution of diffusible mediators (such as BDNF) and axonmicroglia physical contact in the ability of primed microglia to bolster synapse formation.

Overall, these finding show that microglia are more than synaptic strippers, but are actually important controllers of synapse formation. This novel role of microglia on pre-synaptic differentiation may be of particular importance to better understand neurodevelopment disorders characterized by aberrant synapse formation associated with maternal infections during pregnancy.

\section{ACKNOWLEDGMENTS}

This work was supported by PEstC/SAU/LA0001/2013-2014, by FEDER, through Programa Operacional Fatores de Competitividade-COMPETE and by national funds, through FCT-Fundação para a Ciência e a Tecnologia through grants PTDC/SAU-NEU/104100/2008, EXPL/NEU-NMC/0541/2012 and by Marie Curie Actions, International Reintegration Grant, 7th Framework Programme, EU to Ramiro D. Almeida. FCT supports Maria J. Pinto (SFRH/BD/51196/2010) and Catarina Gomes (SFRH/BPD/63013/2009).

\section{REFERENCES}

Benarroch, E. E. (2013). Microglia: multiple roles in surveillance, circuit shaping, and response to injury. Neurology 81, 1079-1088. doi: 10.1212/WNL.0b013e3182a4a577

Bialas, A. R., and Stevens, B. (2013). TGF- $\beta$ signaling regulates neuronal $\mathrm{Clq}$ expression and developmental synaptic refinement. Nat. Neurosci. 16, 1773-1782. doi: 10.1038/nn.3560

Chugh, D., Nilsson, P., Afjei, S. A., Bakochi, A., and Ekdahl, C. T. (2013). Brain inflammation induces post-synaptic changes during early synapse formation in adult-born hippocampal neurons. Exp. Neurol. 250, 176-188. doi: 10.1016/j.expneurol.2013.09.005

Davalos, D., Grutzendler, J., Yang, G., Kim, J. V., Zuo, Y., Jung, S., et al. (2005). ATP mediates rapid microglial response to local brain injury in vivo. Nat. Neurosci. 8, 752-758. doi: 10.1038/nn1472

Gomes, C., Ferreira, R., George, J., Sanches, R., Rodrigues, D. I., Gonçalves, N., et al. (2013). Activation of microglia cells triggers a release of brain-derived neurotrophic factor (BDNF) inducing their proliferation in an adenosine A2A receptor-dependent manner J. Neuroinflammation 10:16. doi: 10.1186/1742-20 94-10-16

Ji, K., Akgul, G., Wollmuth, L. P., and Tsirka, S. E. (2013). Microglia actively regulate the number of functional synapses. PLoS ONE 8:e56293. doi: 10.1371/journal.pone. 0056293

Kettenmann, H., Hanisch, U. K., Noda, M., and Verkhratsky, A. (2011). Physiology of microglia. Physiol. Rev. 91, 461-553. doi: 10.1152/physrev.00011.2010

Kettenmann, H., Kirchhoff, F., and Verkhratsky, A. (2013). Microglia: new roles for the synaptic stripper. Neuron 77, 10-18. doi: 10.1016/j.neuron.2012.12.023

Neto, E., Alves, C. J., Sousa, D. M., Alencastre, I. S., Lourenco, A. H., Leitao, L., et al. (2014). Sensory neurons and osteoblasts: close partners in a microfluidic platform. Integr. Biol. (Camb). doi: 10.1039/c4ib00035h. [Epub ahead of print].

Nimmerjahn, A., Kirchhoff, F., and Helmchen, F. (2005). Resting microglial cells are highly dynamic surveillants of brain parenchyma in vivo. Science 308, 1314-1318. doi: 10.1126/science.11 10647 
Parkhurst, C. N., Yang, G., Ninan, I., Savas, J. N., Yates, J. R. 3rd., Lafaille, J. J., et al. (2013). Microglia promote learning-dependent synapse formation through brain-derived neurotrophic factor. Cell 155, 1596-1609. doi: 10.1016/j.cell.2013. 11.030

Pascual, O., Ben Achour, S., Rostaing, P., Triller, A., and Bessis, A. (2012). Microglia activation triggers astrocyte-mediated modulation of excitatory neurotransmission. Proc. Natl. Acad. Sci. U.S.A. 109, 197-205. doi: 10.1073/pnas.11110 98109

Rogers, J. T., Morganti, J. M., Bachstetter, A. D., Hudson, C. E., Peters, M. M., Grimmig, B. A., et al. (2011). CX3CR1 deficiency leads to impairment of hippocampal cognitive function and synaptic plasticity. J. Neurosci. 31, 16241-16250. doi: 10.1523/JNEUROSCI.3667-11.2011

Schafer, D. P., Lehrman, E. K., Kautzman, A. G., Koyama, R., Mardinly, A. R., Yamasaki, R., et al. (2012). Microglia sculpt postnatal neural circuits in an activity and complementdependent manner. Neuron 74, 691-705. doi: 10.1016/j.neuron.2012.03.026

Schafer, D. P., Lehrman, E. K., and Stevens, B. (2013). The "quad-partite" synapse: microgliasynapse interactions in the developing and mature CNS. Glia 61, 24-36. doi: 10.1002/glia. 22389

Taylor, A. M., Blurton-Jones, M., Rhee, S. W., Cribbs, D. H., Cotman, C. W., and Jeon, N. L. (2005) A microfluidic culture platform for CNS axonal injury, regeneration and transport. Nat. Methods 2, 599-605. doi: 10.1038/nmeth777

Tremblay, M. È., Lowery, R. L., and Majewska, A. K. (2010). Microglial interactions with synapses are modulated by visual experience. PLoS Biol. 8:e1000527. doi: 10.1371/journal.pbio.10 00527

Wake, H., Moorhouse, A. J., Jinno, S., Kohsaka, S., and Nabekura, J. (2009). Resting microglia directly monitor the functional state of synapses in vivo and determine the fate of ischemic terminals. J. Neurosci. 29, 3974-3980. doi: 10.1523/JNEUROSCI.4363-08.2009

Zhan, Y., Paolicelli, R. C., Sforazzini, F., Weinhard, L., Bolasco, G., Pagani, F., et al. (2014). Deficient neuron-microglia signaling results in impaired functional brain connectivity and social behavior. Nat. Neurosci. 17, 400-406. doi: 10.1038/ nn.3641

Zhang, J., Malik, A., Choi, H. B., Ko, R. W., Dissing-Olesen, L., and Macvicar, B. A. (2014). Microglial CR3 activation triggers long-term synaptic depression in the hippocampus via NADPH oxidase. Neuron 82, 195-207. doi: 10.1016/j.neuron.2014.01.043

Conflict of Interest Statement: The authors declare that the research was conducted in the absence of any commercial or financial relationships that could be construed as a potential conflict of interest.

Received: 30 April 2014; accepted: 13 May 2014; published online: 02 June 2014.

Citation: Cristovão G, Pinto MJ, Cunha RA, Almeida $R D$ and Gomes CA (2014) Activation of microglia bolsters synapse formation. Front. Cell. Neurosci. 8:153. doi: 10.3389/fncel.2014.00153

This article was submitted to the journal Frontiers in Cellular Neuroscience.

Copyright (C) 2014 Cristovão, Pinto, Cunha, Almeida and Gomes. This is an open-access article distributed under the terms of the Creative Commons Attribution License (CC BY). The use, distribution or reproduction in other forums is permitted, provided the original author(s) or licensor are credited and that the original publication in this journal is cited, in accordance with accepted academic practice. No use, distribution or reproduction is permitted which does not comply with these terms. 